Dominik Grether, Benjamin Kickhöfer, Kai Nagel

\title{
Policy Evaluation in Multiagent Transport Simulations
}

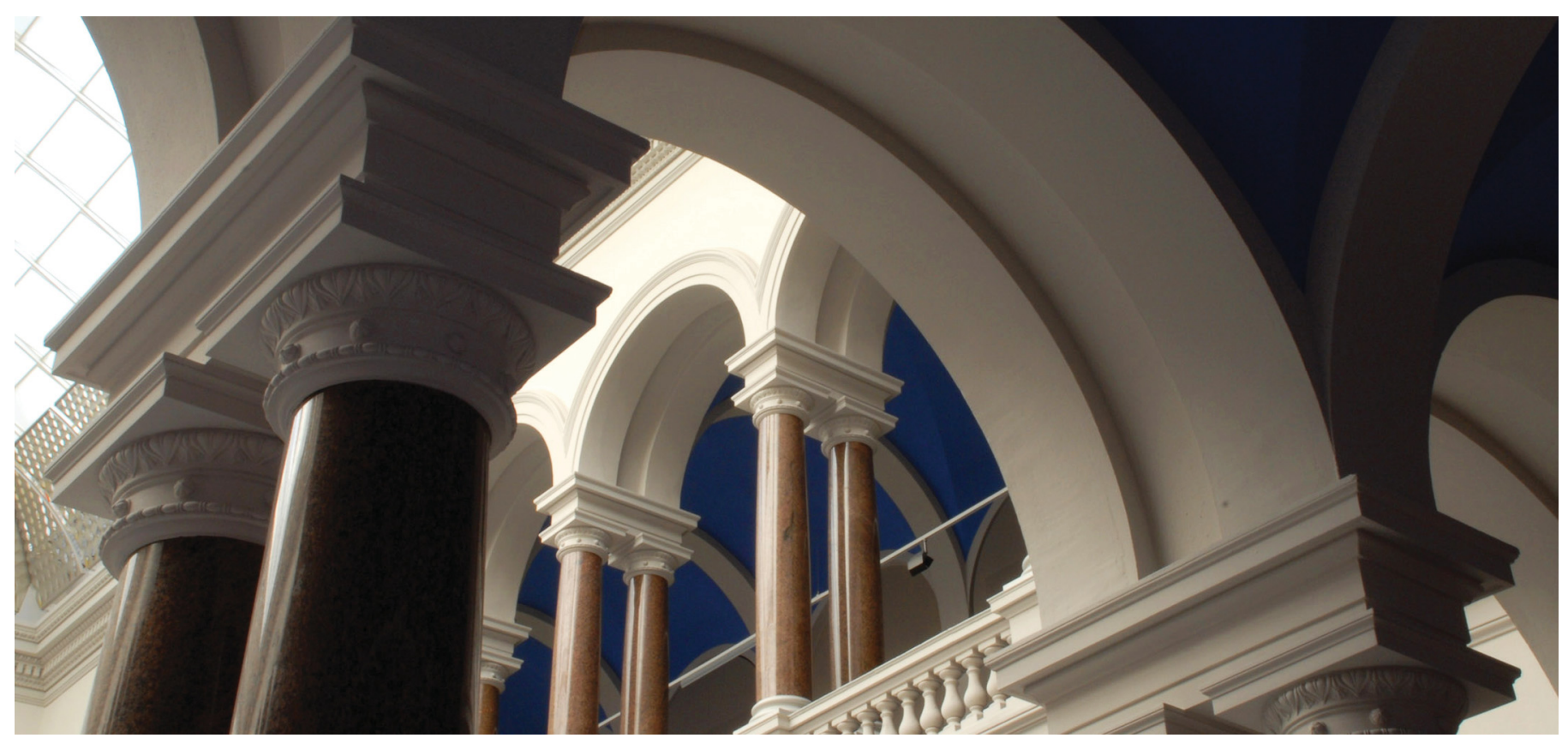

Dominik Grether, Benjamin Kickhöfer, Kai Nagel, Policy Evaluation in Multiagent Transport Simulations, Transportation Research Record: Journal of the Transportation Research Board (Vol 2175, Issue 1) pp. 10-18. Copyright (C) [2010] (Sage). DOI: [10.3141/2175-02]. 
Policy evaluation in multi-agent transport simulations

2009-nov-15

Dominik Grether

Transport Systems Planning and Transport Telematics, Berlin Institute of Technology, D-10587 Berlin

phone: +49-30-314 21383

fax: +49-30-314 26269

grether@vsp.tu-berlin.de

Benjamin Kickhöfer

Transport Systems Planning and Transport Telematics, Berlin Institute of Technology, D-10587 Berlin

phone: +49-30-314 23308

fax: +49-30-314 26269

kickhoefer@vsp.tu-berlin.de

Kai Nagel

Transport Systems Planning and Transport Telematics, Berlin Institute of Technology, D-10587 Berlin

phone: +49-30-314 23308

fax: +49-30-314 26269

nagel@vsp.tu-berlin.de

Words: $6937+2$ figures 


\begin{abstract}
In democratically organized societies, the implementation of measures with regressive effects on the welfare distribution tends to be complicated due to low public acceptance. The microscopic multi-agent simulation approach presented in this paper is capable to help designing better solutions in such situations. It is shown that income can be included in utility calculations for a better understanding of problems linked to acceptability. This paper shows how the approach can be used in policy evaluation when including income in the user preferences. Using the MATSim framework, the implementation is tested in a simple scenario. Furthermore, and going beyond (1), it is shown that the approach works in a large-scale real world example. Based on a hypothetical price and speed increase of public transit, effects on the welfare distribution of the population are discussed. It is shown that this approach, in contrast to applied economic policy analysis, allows choice modeling and economic evaluation to be realised in a consistent way.
\end{abstract}




\section{INTRODUCTION}

Policy measures in transportation planning aim at improving the system as a whole. Changes to the system that result in an unequal distribution of the overall welfare gain are, however, hard to implement in democratically organized societies. Studies indicate that, e.g., tolls tend to be regressive if no redistribution scheme is considered at the same time, and may so increase the inequality in welfare distribution (1). An option to reach broader public acceptance for such policies may be to include the redistribution of total gains into the scheme. Hence, methods and tools are needed that simulate welfare changes due to policies on a highly granulated level, i.e. considering each individual of the society. With such tools, policy makers are able to consider impacts of different proposed measures on the welfare distribution. In addition, it is possible to estimate the support level within the society and, if necessary, to evaluate alternatives for further discussion.

Traditional transport planning tools using the four-step process combined with standard economic appraisal methods (e.g. 2) are not able to provide such analysis. In order to bridge this gap, multi-agent microsimulations can be used. Large-scale multi-agent traffic simulations are capable of simulating complete day-plans of several millions of individuals (agents) (3). In contrast to traditional models, all attributes that are attached to the synthetic travelers are kept during the simulation process, thus enabling highly granulated analysis (4). Being aware of all attributes enables the possibility to attach to every traveller an individual utility function that is used to maximize the individual return of travel choices during the simulation process. Another advantage of the multi-agent simulation technique is the connection of travelers' choices along the time axis when simulating time dependent policies (5).

In the context of policy evaluation, simulation results can immediately be used to identify winners and losers, since the utility scores of the individual agents are kept and can be compared between scenarios agent-by-agent. They can also be aggregated in arbitrary ways, based on any available demographic attributes including spatial information of high resolution. Welfare computations, if desired, can be done on top of that, without having to resort to indirect measures such as link travel times or inter-zonal impedances. The usual problems when monetarizing the individual utility still apply (6), but at least one of the main issues in applied economic analysis is addressed: with multi-agent approaches, choice modeling and economic evaluation are implemented in a consistent framework, similar to efforts to base such analysis directly on discrete choice models (7).

This paper shows how multi-agent approaches can be used in policy evaluation adding an individual income attribute to each agent so that personalized utilities are considered. It is shown how one benefits doing so when considering issues linked with public acceptance. Implications on the simulation model are explained and the measurement of welfare effects resulting from policy measures is focused. In a simple scenario income is the only varying attribute between the agents. A real world scenario, however, includes varying trip distances and day plans so that demographic attributes of each agent are strongly personalized.

The paper is organized as follows: First, the simulation structure is introduced. Then, the income-dependent utility function used in this paper is presented. Afterwards, in a simple scenario, the correctness of implementation and the plausibility of results is tested. Subsequently, a realistic simulation of regular workday traffic in the Zurich metropolitan area is performed, including the effects of a public transit price and speed increase. Afterwards, welfare changes across the income range and open issues are discussed. The paper ends with a conclusion. 


\section{SIMULATION STRUCTURE}

The following describes the structure of the simulation that is used. It is the standard structure of MATSim $^{1}$, as described at many places $(8,9)$. Readers familiar with the MATSim approach can skip this section.

\section{Overview}

In MATSim, each traveler of the real system is modeled as an individual agent. The overall approach consists of three important parts:

- Each agent independently generates a so-called plan, which encodes its preferences during a certain time period, typically a day.

- All agents' plans are simultaneously executed in the simulation of the physical system, the so called traffic flow simulation or mobility simulation.

- There is a mechanism that allows agents to learn. In the implementation, the system iterates between plans generation and traffic flow simulation. The system remembers several plans per agent, and scores the performance of each plan. Agents normally choose the plan with the highest score, sometimes re-evaluate plans with bad scores, and sometimes obtain new plans by modifying copies of existing plans.

A plan contains the itinerary of activities that the agent wants to perform during the day, plus the intervening trip legs the agent must take to travel between activities. An agent's plan details the order, type, location, duration and other time constraints of each activity, and the mode, route and expected departure and travel times of each leg.

A plan can be modified by various modules. In the test scenario, the Time Adaptation module is used, while the large-scale application additionally uses a Router module. The Time Adaptation module changes the timing of an agent's plan. A very simple approach is used which just applies a random "mutation" to the duration attributes of the agent's activities (9). The Router is a timedependent best path algorithm (10), using the the link's generalised costs based on the link travel times of the previous iteration. Mode choice will not be simulated by a module per se, but instead by making sure that every agent has at least one "car" and at least one "public transit" plan (11).

One of the plans of every agent is marked as "selected". The traffic flow simulation executes all agents' selected plans simultaneously on the network and provides output describing what happened to each individual agent during the execution of its plan. The car traffic flow simulation is implemented as a queue simulation, where each street (link) is represented as a first-in first-out queue with two restrictions $(12,13)$ : First, each agent has to remain for a certain time on the link, corresponding to the free speed travel time. Second, a link storage capacity is defined which limits the number of agents on the link; if it is filled up, no more agents can enter this link. The public transit simulation simply assumes that travel by public transit takes twice as long as travel by car on the fastest route in an empty network (11), and that the travel distance is 1.5 times the beeline distance. Public transit is assumed to run continuously and without capacity restrictions.

The modules base their decisions on the output of the traffic flow simulation (e.g. knowledge of congestion) using feedback from the multi-agent simulation structure $(14,15)$. This sets up an iteration cycle which runs the traffic flow simulation with specific plans for the agents, then uses the planning modules to update the plans; these changed plans are again fed into the traffic flow

\footnotetext{
${ }^{1}$ Multi-Agent Transport Simulation, see www.matsim.org
} 
simulation until consistency between the modules is reached. The feedback cycle is controlled by the agent database, which also keeps track of multiple plans generated by each agent.

In every iteration, $10 \%$ of the agents generate new plans by taking an existing plan, making a copy of it, and then modifying the copy with the Time Adaptation or the Router module. The other agents reuse one of their existing plans. The probability to change the selected plan is calculated according to

$p_{\text {change }}=\min \left(1, \alpha \cdot e^{\beta \cdot\left(s_{\text {random }}-s_{\text {current }}\right) / 2}\right)$,

where

- $\alpha$ : The probability to change if both plans have the same score, set to $1 \%$

- $\beta$ : A sensitivity parameter, set to 20 for the tests and to 2 for the large-scale Zurich simulations

- $s_{\{\text {random,current }\}}$ : The score of the current/random plan (see later)

In the steady state, this model is equivalent to the standard multinomial logit model $p_{j}=\frac{e^{\beta \cdot s_{j}}}{\sum_{i} e^{\beta \cdot s_{i}}}$, where $p_{j}$ is the probability for plan $j$ to be selected.

The repetition of the iteration cycle coupled with the agent database enables the agents to improve their plans over many iterations. As the number of plans is limited for every agent by memory constraints, the plan with the worst performance is deleted when a new plan is added to a person which already has the maximum number of plans permitted. The iteration cycle continues until the system has reached a relaxed state. At this point, there is no quantitative measure of when the system is "relaxed"; we just allow the cycle to continue until the outcome is stable.

\section{Scoring Plans}

In order to compare plans, it is necessary to assign a quantitative score to the performance of each plan. In this work, in order to be consistent with economic appraisal, a simple utility-based approach is used. The approach is related to the Vickrey bottleneck model $(16,17)$, but is modified in order to be consistent with our approach based on complete daily plans $(18,19)$. The elements of our approach are as follows:

- The total score ${ }^{2}$ of a plan is computed as the sum of individual contributions:

$$
U_{\text {total }}=\sum_{i=1}^{n} U_{\text {perf }, i}+\sum_{i=1}^{n} U_{\text {late }, i}+\sum_{i=1}^{n} U_{t r, i},
$$

where $U_{\text {total }}$ is the total utility for a given plan; $n$ is the number of activities, which equals the number of trips (the first and the last activity are counted as one); $U_{\text {perf }, i}$ is the (positive) utility earned for performing activity $i$; $U_{\text {late }, i}$ is the (negative) utility earned for arriving late to activity $i$; and $U_{t r, i}$ is the (usually negative) utility earned for traveling during trip $i$.

- A logarithmic form is used for the positive utility earned by performing an activity:

$$
U_{\text {perf }, i}\left(t_{\text {perf }, i}\right)=\beta_{\text {perf }} \cdot t_{*, i} \cdot \ln \left(\frac{t_{p e r f, i}}{t_{0, i}}\right)
$$

\footnotetext{
${ }^{2}$ Note that the terms "score" and "utility" refer to the same absolute value. "Utility" is the common expression in economic evaluation and is therefore used in this paper.
} 
where $t_{\text {perf }}$ is the actual performed duration of the activity, $t_{*}$ is the "typical" duration of an activity, and $\beta_{\text {perf }}$ is the marginal utility of an activity at its typical duration. $\beta_{\text {perf }}$ is the same for all activities, since in equilibrium all activities at their typical duration need to have the same marginal utility.

$t_{0, i}$ is a scaling parameter that is related both to the minimum duration and to the importance of an activity. As long as dropping activities from the plan is not allowed, $t_{0, i}$ has no effect.

- The (dis)utility of being late is uniformly assumed as:

$U_{\text {late }, i}\left(t_{\text {late }, i}\right)=\beta_{\text {late }} \cdot t_{\text {late }, i}$,

where $\beta_{\text {late }}$ is the marginal utility (in $1 / \mathrm{h}$ ) for being late, and $t_{\text {late } i}$ is the number of hours late to activity $i$. $\beta_{\text {late }}$ is usually negative.

- The (dis)utility of traveling used in this paper is estimated from survey data, illustrated in (20). It is assumed to be dependent on the transport mode and the individual income as explained in the following section.

In principle, arriving early could also be punished. There is, however, no immediate need to punish early arrival, since waiting times are already indirectly punished by foregoing the reward that could be accumulated by doing an activity instead (opportunity cost). In consequence, the effective (dis)utility of waiting is already $-\beta_{\text {perf }} t_{*, i} / t_{\text {perf }, i} \approx-\beta_{\text {perf }}$. Similarly, that opportunity cost has to be added to the time spent traveling.

No opportunity cost needs to be added to late arrivals, because the late arrival time is spent somewhere else. In consequence, the effective (dis)utility of arriving late remains at $\beta_{\text {late }}$.

\section{INCOME-DEPENDENT UTILITY FUNCTION}

There is some agreement that income effects play an important role in transport policy analysis, see, e.g., $(21,22,23,6,1)$. The argument essentially is that monetary price changes affect different income groups differently. This is usually addressed by including income dependent user preferences in the utility function.

\section{Functional Form}

The functional form used for simulations is loosely based on (1) and is similar to (24). A detailed derivation of this form and the estimation of the corresponding parameters are illustrated in (20). Hence, the utility functions of the two transport modes car and public transit (pt) are, according to (2), given by:

$$
\begin{aligned}
& U_{c a r, i, j}=+\frac{1.86}{h} t_{*, i} \cdot \ln \left(\frac{t_{\text {perf }, i}}{t_{0, i}}\right) \quad-\frac{1.52}{h} t_{\text {late }, i}-4.58 \frac{c_{i, c a r}}{y_{j}} \quad-\frac{0.97}{h} t_{i, c a r} \\
& U_{p t, i, j}=+\frac{1.86}{h} t_{*, i} \cdot \ln \left(\frac{t_{p e r f, i}}{t_{0, i}}\right)-\frac{1.52}{h} t_{\text {late }, i}-4.58 \frac{c_{i, p t}}{y_{j}}
\end{aligned}
$$

The first summand refers to (3) with $\beta_{\text {perf }, i}=+1.86 / h$, the second summand corresponds with (4) and $\beta_{\text {late }, i}=+1.52 / h$. The third and forth summands introduce mode and income dependency to the utility functions: $y_{j}$ stands for the daily income of person $j$ and $c_{i}$ is monetary cost for the trip to activity $i$. The indices car and $p t$ indicate the mode. Trip costs are calculated using $c_{i, c a r}=$ $0.12 \mathrm{CHF} / \mathrm{km}$ and $c_{i, p t}=0.28 \mathrm{CHF} / \mathrm{km}$. While there is a forth summand for car $\left(\beta_{t t, c a r}=\right.$ 
$-0.97 / h)$, picking up the linear disutility of travel time $t_{i}$, there is no equivalent expression in the pt utility function. Travel time in pt is nonetheless punished by the opportunity costs of time by missing out on positive utility of an activity $\left(\beta_{\text {perf }, i}\right)$ which also implies additional negative utility for the car travel time.

The individual income is derived for each agent based on several Lorenz curves. Methodical details can be found in (20) also showing that a distribution similar to reality is generated. Utilities are computed in "utils"; a possible conversion into units of money or "hours of leisure time" (25) needs to be done separately (see "Discussion"). Note that late arrival parameter will only be used in the test but not in the real-world scenario.

\section{TEST SCENARIO}

The goal of this section is to verify the correctness and plausibility of the estimated choice model and the underlying implementation. Since probabilistic multi-agent simulations and other software systems tend to be sensitive to new implementations, a simple setup is used in order to test the plausibility of traveler choice reactions as a result of a fictive policy change.

\section{Network}

The test network consists of a cycle of one-way links with (unrealistically) high capacities so as to minimize their influence on traffic patterns, essentially making it possible for most agents to drive with free speed. One link between home and work location has reduced capacity of $1000 \mathrm{veh} / \mathrm{h}$, building a bottleneck.

\section{Initial Plans}

The synthetic population consists of 2000 agents. All agents start at their home activity, which they initially leave at 06:00. They initially drive to work with a car, stay there for 8 hours and drive home afterwards. The home to work trip has a length of $17.5 \mathrm{~km}$ while the way back is $32.5 \mathrm{~km}$ long. Speed limit is at $50 \mathrm{~km} / \mathrm{h}$ so the free speed travel time from home to work by car is 21 minutes while 39 minutes are needed for the way back home. Thus the total free speed travel time driving by car is 60 minutes. As the agents are forced to remain on that route, the scenario is similar to the well-known Vickrey bottleneck scenario $(17,16)$; also see below for more details.

In addition, each agent possesses an initially non-active plan that uses the public transit mode for both trips. These trips take twice as long as by car at freespeed, i.e. 42 minutes from home to work, and 78 minutes for the way back. The total public transit travel time is 120 minutes. In contrast to the car travel times, these transit travel times are not affected by congestion. Since public transit is assumed to run continuously and without capacity restrictions, a home departure at time $t$ will always result in a work arrival at $t+42 \mathrm{~min}$.

Work opens at 07:00 am and closes at 06:00 pm. In order to obtain the similarity to the Vickrey scenario, an additional behavioral parameter of $\beta_{\text {late }}=-1.52 / \mathrm{h}$ is used, i.e. deducting $-1.52 / \mathrm{h}$. $t_{\text {late }}$ for arriving late. In order to be consistent with the Vickrey bottleneck scenario, any arrival time after 07:00 am is directly considered as late.

Estimation of income for the synthetic population is based on data from Kanton Zurich. ${ }^{3}$ Income distribution is retrieved from a Lorenz curve for the year $2006 .{ }^{4}$ The median income for that

\footnotetext{
${ }^{3}$ http: //www.statistik.zh. ch/themenportal/themen/index.php, last access 01.08.2009

${ }^{4}$ http://www.statistik.zh.ch/themenportal/themen/aktuell_detail.php?id= $2752 \& t b=4 \& m t=0$, last access 01.08.2009
} 
year is $46300 \mathrm{CHF}$.

\section{Behavioral Parameters}

The behavioral parameters are set and can be interpreted as follows:

- marginal utility of performing an activity at its typical duration: $\beta_{\text {perf }}=1.86 / \mathrm{h}$

- marginal disutility of arriving late: $\beta_{\text {late }}=-1.52 / \mathrm{h}$

- marginal utility offset for traveling with a car: $\beta_{t r, c a r}=-0.97 / h$

- marginal utility offset for traveling with public transit mode: $\beta_{p t}=0$

- factor in logit process (eq. 1): $\beta=20$

- "typical" durations of $t_{*, w}=8$ and $t_{*, h}=12$ hours for work and home mean that work and home times have a tendency to arrange themselves with a ratio of 8:12 (i.e. 2:3).

The activity of the home activity is "wrapped around", i.e. a departure at 6am and a return at $5 \mathrm{pm}$ results in a home activity duration of 13 hours.

A work start exactly at 7:00am means that (a) no utility can be accumulated from an arrival earlier than 7:00am, and (b) any late arrival is immediately punished with $\beta_{\text {late }}=-1.52 / \mathrm{h}$.

Because of the argument made earlier regarding the opportunity cost of foregone activity time when arriving early, the effective marginal disutility of early arrival is $\beta_{\text {early,eff }}=$ $-\beta_{\text {perf }} t_{*, i} / t_{\text {perf }, i} \approx-\beta_{\text {perf }}=-1.86 / h$ which is equal to the effective marginal disutility of traveling with public transit $\beta_{t t_{p t}, \text { eff }}$. The effective marginal disutility of traveling with car is, by the same argument, $\beta_{t t_{c a r}, \text { eff }}=-\beta_{\text {perf }} t_{*, i} / t_{\text {perf }, i}-\left|\beta_{t t_{c} a r}\right| \approx-\beta_{\text {perf }}-\left|\beta_{t t_{c} a r}\right| \approx-2.83 / h$. The return trip has no influence since there is no congestion.

Overall, the effective values of car travel time of our study would correspond to the values $\left(\beta_{\text {early,eff }}, \beta_{t t_{c a r}, \text { eff }}, \beta_{\text {late eff }}\right)=(-1.86,-2.83,-1.52)$ of the Vickrey scenario $(16,17)$.

\section{Simulation Runs}

First, a "preparatory run" - referred to as base case - is performed for 4000 iterations. During the first 2000 iterations, $10 \%$ of the agents perform "time adaptation", i.e. they make a copy of an existing plan and shift each element of its time structure by a random amount between zero and 7.5 minutes. The other $90 \%$ of the agents switch between their existing plans according to (1), which means that they potentially also switch the mode. During the second 2000 iterations, time adaptation is switched off; in consequence, agents only switch between existing plans according to (1). That is, their choice set now remains fixed to what they have found in the first 2000 iterations, and they choose within this set according to a logit model.

After this, the policy measure is introduced. Every policy case is run for another 2000 iterations, starting from the final iteration of the preparatory run. For the first 1000 iterations of the policy case, the time adaption module is again switched on, with the same $10 \%$ replanning fraction. The final 1000 iterations are once more with a fixed choice set. The following three different policy measures are simulated:

- public transit price increase: The price of public transit, i.e. $c_{i, p t}$, is raised by $20 \%$ from 0.28 to $0.336 \mathrm{CHF} / \mathrm{km}$.

- public transit speed increase: The speed of public transit is increased, now taking only 1.8 (instead of 2.0) times as long as the freespeed car. This equals a speed increase of $10 \%$.

- combined: Combination of the two measures above. 
The policy design is based the estimation about price and travel time elasticities (26). In this collection of different studies, travel time elasticities are identified to be double as high as price elasticities. Thus, for the combined policy measure, one would expect almost no shift in the modal split. In the following section, iteration 4000 of the base case is then compared to the final iteration of the combined measure. For reasons of clarity, results of the pure pt price increase and of the pure pt speed increase are not discussed in this paper. A detailed analysis can be found in (20).

\section{Results}

According to Fig. 1a, low income people predominantly use the car while high income people predominantly use public transit. This means that car is the low value and public transit the high value mode. Even though a bit surprising, this is consistent with the higher costs of $c_{p t}=0.28 \mathrm{CHF} / \mathrm{km}$ assumed for public transit than for car $\left(c_{c a r}=0.12 \mathrm{CHF} / \mathrm{km}\right)$, which were used for parameter estimation $(27,24,20)$. The overall modal split of the base case is $54 \%: 46 \%$ (car:pt).

The combined measure leads to a modal split of 44\%:56\% (car:pt). Obviously, $10 \%$ of the agents change from car to pt. This happens through a shift of the income level that divides the two regimes: it moved towards lower incomes (Fig. 1b).

Generally speaking, this means that people react more sensitive to the speed than to the price increase. These results are contradictory to the expectations based on (26) that predict an roughly unchanged modal split.

Fig. 1c shows, agent-by-agent, the utility differences between the base case and the policy case as a scatter plot over deciles of the population. Every decile contains the same number of agents, sorted by their income. The four different colors in the plots correspond to four different user groups that can be identified as a result of the policy change. Red quads and green dots represent agents that choose the same transport mode before and after the measure, red for the car mode, green for the pt mode. Blue triangles and yellow trapezoids stand for agents that change their transport mode, where blue means a change from pt to car and yellow a switch from car to pt. For analysis purposes, mean values of utility change are computed for every group within the population deciles. A threshold of 4 was used for the plot, meaning that population deciles with less than four agents in the corresponding group were not taken into consideration.

At a first glance, one notices a "fan" of points at lower income levels, and a more correlated structure stretching from middle to higher income levels. The "fan" can be traced back to the car users, who, because of stochastic congestion effects, face rather strong fluctuations of their utilities. In contrast, the correlated structure is caused by the pt users.

As Fig. 1c nicely shows, the measure leads to an overall utility gain across all income and user groups. First, public transit users predictably lose from the price increase, but simultaneously gain from the speed increase. The price increase component affects lower income people more than higher income people, while the inverse is true for the speed increase. Second, a few mid income people change from pt to car. Thus, for them the negative utility changes from the price increase overweight the travel time savings from the speed incease. Third, with increasing income the pt mode becomes more attractive because travel time savings get more important than the additional pt fee. Last, for car users, the pt price increase leads to an increased car share, thus more car congestion, thus utility losses for car users; the speed increase leads to a reduced car share, thus less car congestion, thus utility gains for car users. In average the car users still gain which means that the global effect of the pt speed increase is dominating.

Note that the second and the third effect are strongly influenced by stochastic effects in the plan 


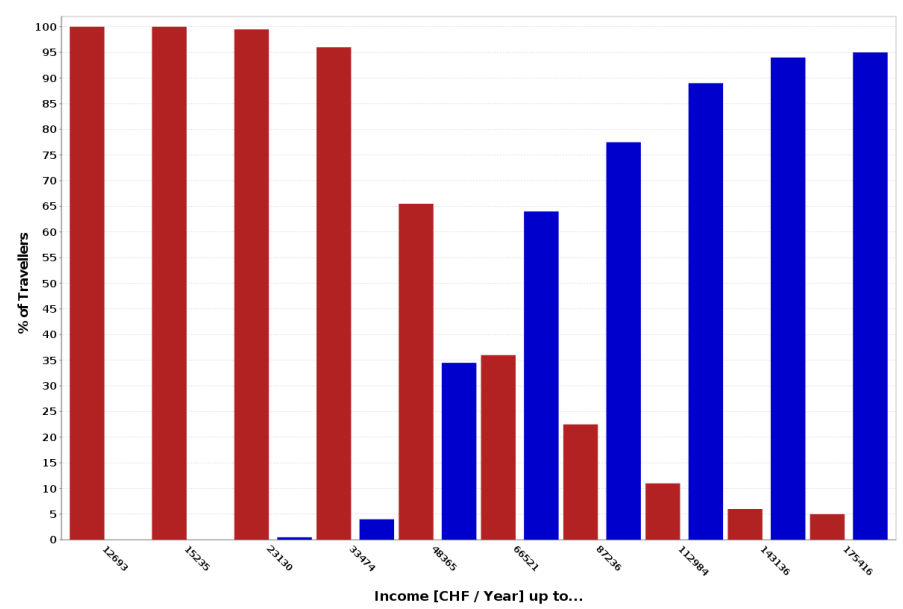

(a) Base case: modal split

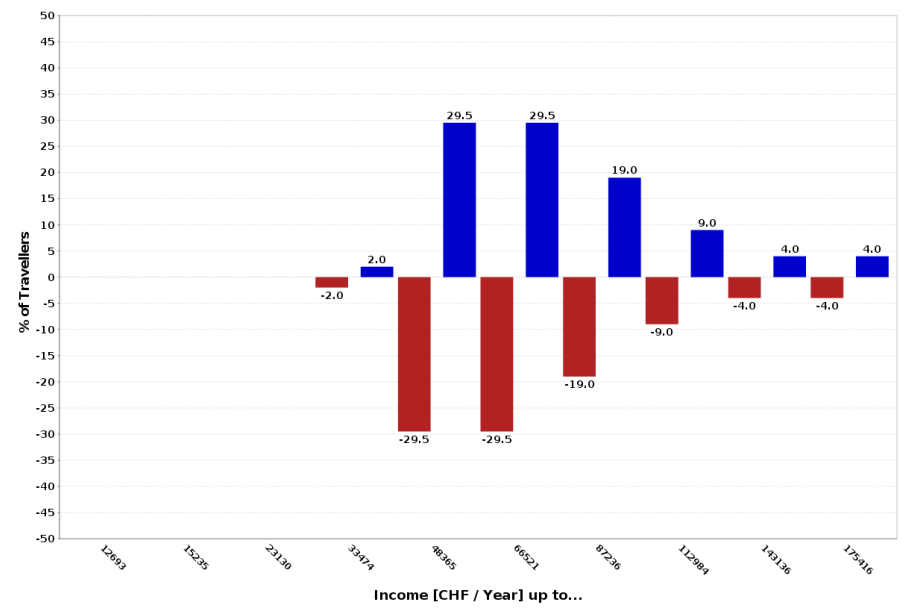

(b) Combined measure: modal split

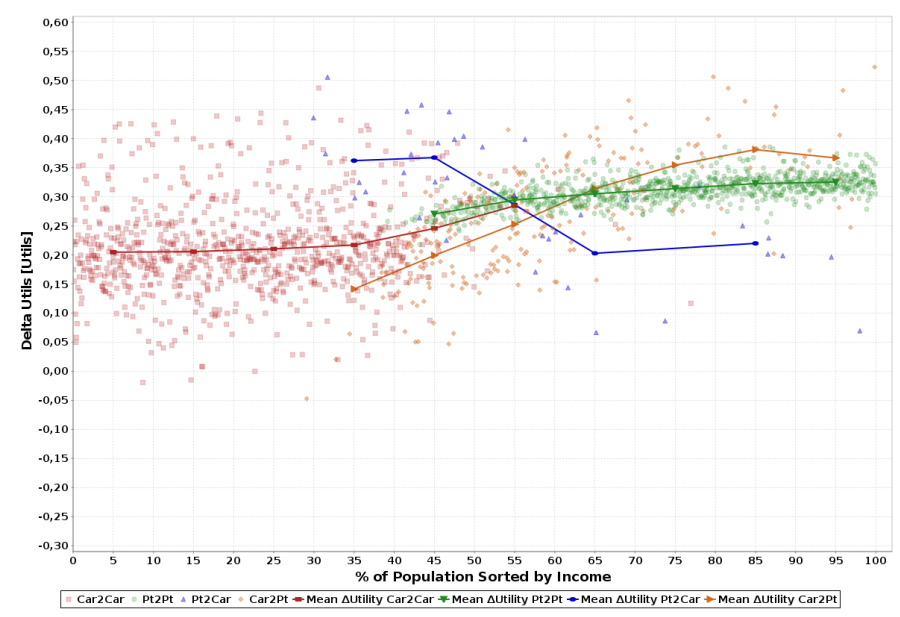

(c) Combined measure: utility changes

FIGURE 1 Results Test Scenario: (a) \& (b) Modal split over decils of the population sorted by income; Red bars depict car drivers, blue bars public transit users (a) Base case: absolute values (b) Combined measure: changes in percentage points (c) Combined measure: utility changes per per person and mode; average utility changes per population decile sorted by income 
selection process (see section "Simulation Structure, Overview"): Mid income agents that did, in the base case, randomly choose a pt plan instead of a better car plan, get on a higher utility level when changing to car after the policy only because of stochastic effects. At higher income levels, agents that, in the policy case, randomly choose a car plan instead of a better pt plan get on a lower utility level for the same reason. Vice versa, the same is true for the yellow curve.

Overall, the results demonstrate that the approach picks up the distributional effects of transport policy measures. While both price and quality-of-service changes affect mode share, achieving this with price changes affects the lower income groups more, while achieving this with qualityof-service changes affect the higher income groups more. Thus, these plausibility tests can be regarded as successful. The approach is therefore applied to a real-world scenario of Zurich metropolitan area in the next section. 


\section{SCENARIO - ZURICH SWITZERLAND}

The income-dependent utility function is now applied to a large-scale, real-world scenario. We use the area of Zurich, Switzerland, which counts about 1 million inhabitants. The following paragraphs give a simplified description of the scenario a full description of the scenario and a calibration analysis can be found in $(28,20)$.

\section{Network and Population}

The network is a Swiss regional planning network that includes the major European transit corridors. It consists of 24180 nodes and 60492 links.

The simulated demand consists of all travelers within Switzerland that are inside an imaginary $30 \mathrm{~km}$ boundary around Zurich at least once during their day All agents have complete day plans with activities like home, work, education, shopping, leisure, based on microcensus information $(29,30)$. The time window during which activities can be performed is limited to certain hours of the day: work and education can be performed from 07:00 to 18:00, shopping from 08:00 to 20:00, while home and leisure have no restrictions. Each agent gets two plans based on the same activity pattern. The first plan uses only "car" as transportation mode, while the second plan uses only public transit.

Unlike the test scenario described above, there is no punishment for being late.

To speed up computations, a random $10 \%$ sample is taken from the synthetic population for simulation, consisting of 181725 agents. In this large-scale application, the agents can, in addition to the time adaptation, also perform route adaptation, which is essential for the car mode. Also mode adaptation is implicitly included (see "Simulation Structure, Overview").

\section{Simulation Runs}

In order to maintain consistency with the test scenarios, the total amount of iterations is reduced but the proportion of the different simulation steps is held constant. This means for the base case:

- For 1000 iterations, $10 \%$ of the agents perform "time adaptation" and 10\% adapt routes (see "Simulation Structure"). The rest of the agents switch between their existing plans, which implicitly includes mode choice.

- During the second 1000 iterations, time and route adaption are switched off; in consequence, agents only switch between existing options.

After this, the policy measure is introduced and is run for another 1000 iterations, starting from the final iteration of the base case. Again, during the first 500 iterations $10 \%$ of the agents perform "time adaptation" while another $10 \%$ of agents adapt routes. Agents neither adapting time nor route switch between existing plans and such eventually switch between transport modes. For the final 500 iterations only a fixed choice set is available.

Different paramter combinations were tested, up to an overall $30 \%$ public transit speed increase and a $60 \%$ raise of public transit prices.

In order to evaluate the impact of the policy, iteration 2000 of the base case is now compared to the final iteration of the combined measure. Similar to the measure in the test scenario public transit price is raised by $10 \%$ and speed is increased by $20 \%$.

\section{Results}

The base case exhihits a modal split of 60.9\%:39.1\% (car:pt). Fig. 2a depicts the modal split in the income deciles of the population. In contrast to the base case of the test scenario shown in Fig. 1a, 
the distribution here is more homogeneous. Both modes are used across all deciles. The highest percentage of car users can be observed from the 3rd to 5th decile whereas in the test scenario this is from the 1 st to 3 rd decile.

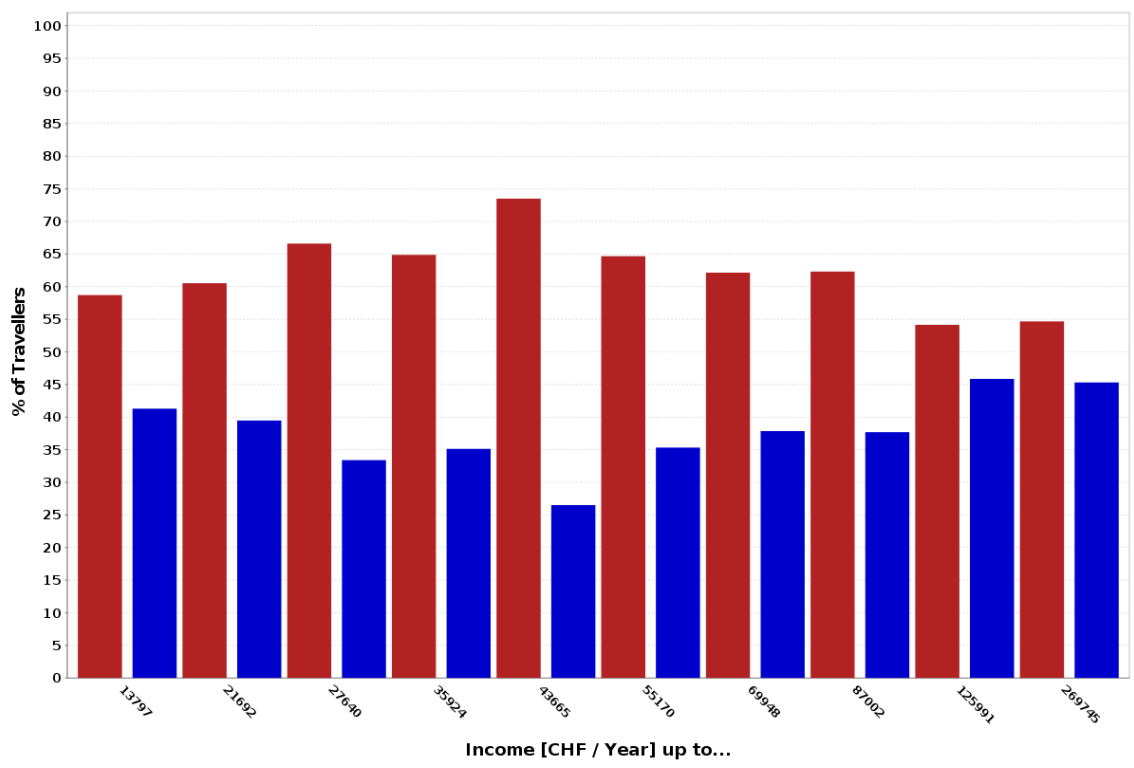

(a) Base case: modal split

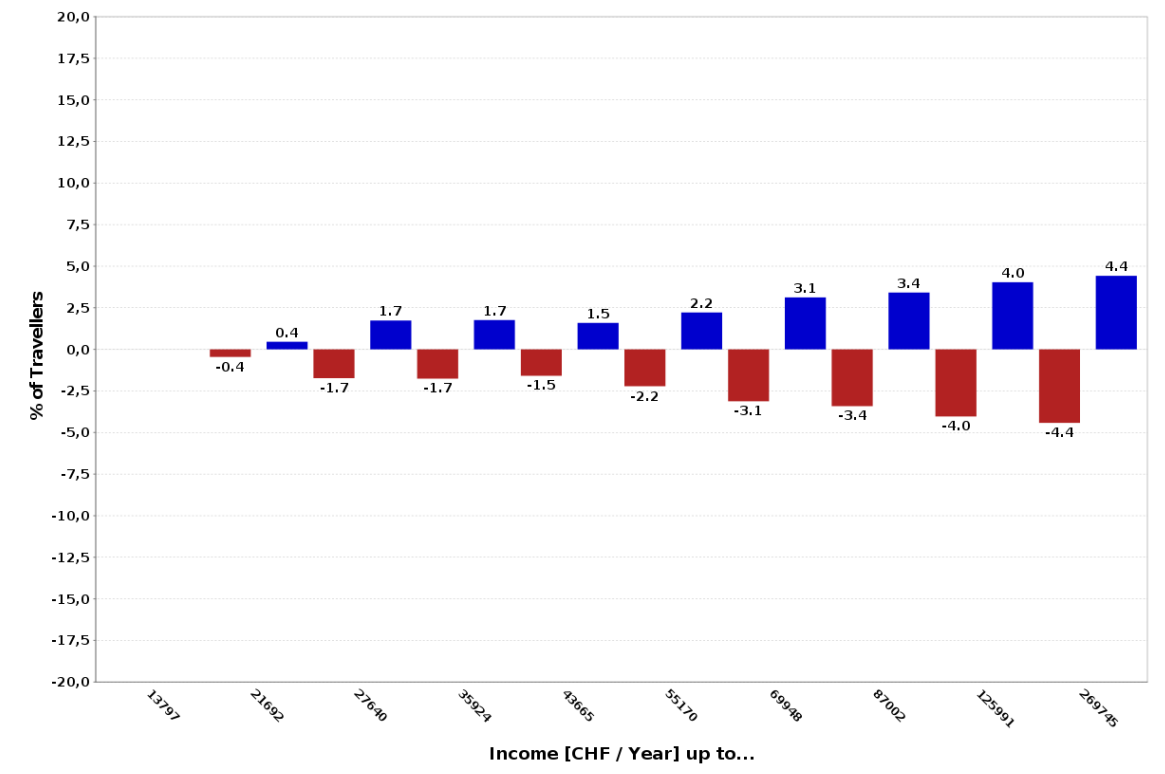

(b) Combined measure: modal split

FIGURE 2 Results Zurich Scenario: Modal Split over income deciles. Red bars depict car drivers, blue bars public transit users. (a) Base Case (b) Difference Combined Measure vs Base Case

The combined measure for public transit results in a mode share of 58.5\%:41.5\% (car:pt). Due to the speed and price increase of the pt, in total $2.4 \%$ of car travellers change from car to pt. Fig. $2 \mathrm{~b}$ presents changes to the modal split in the income decils of population compared to the base case. 


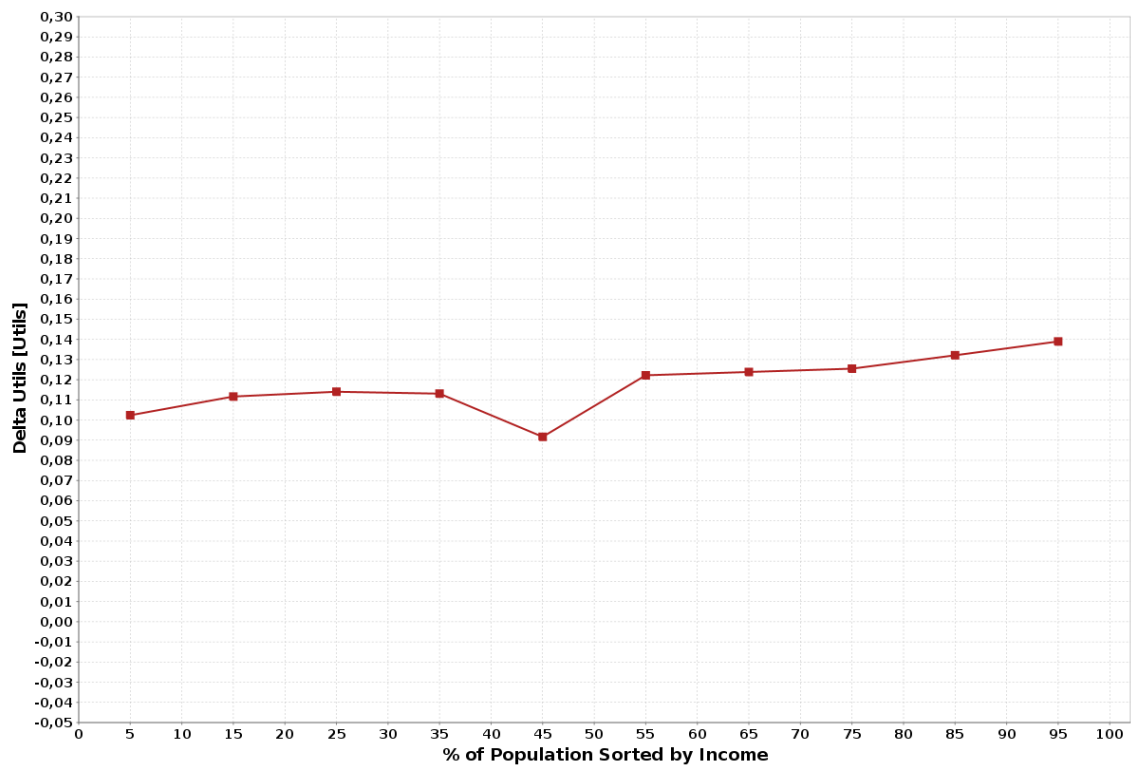

(c) Combined measure: average utility changes

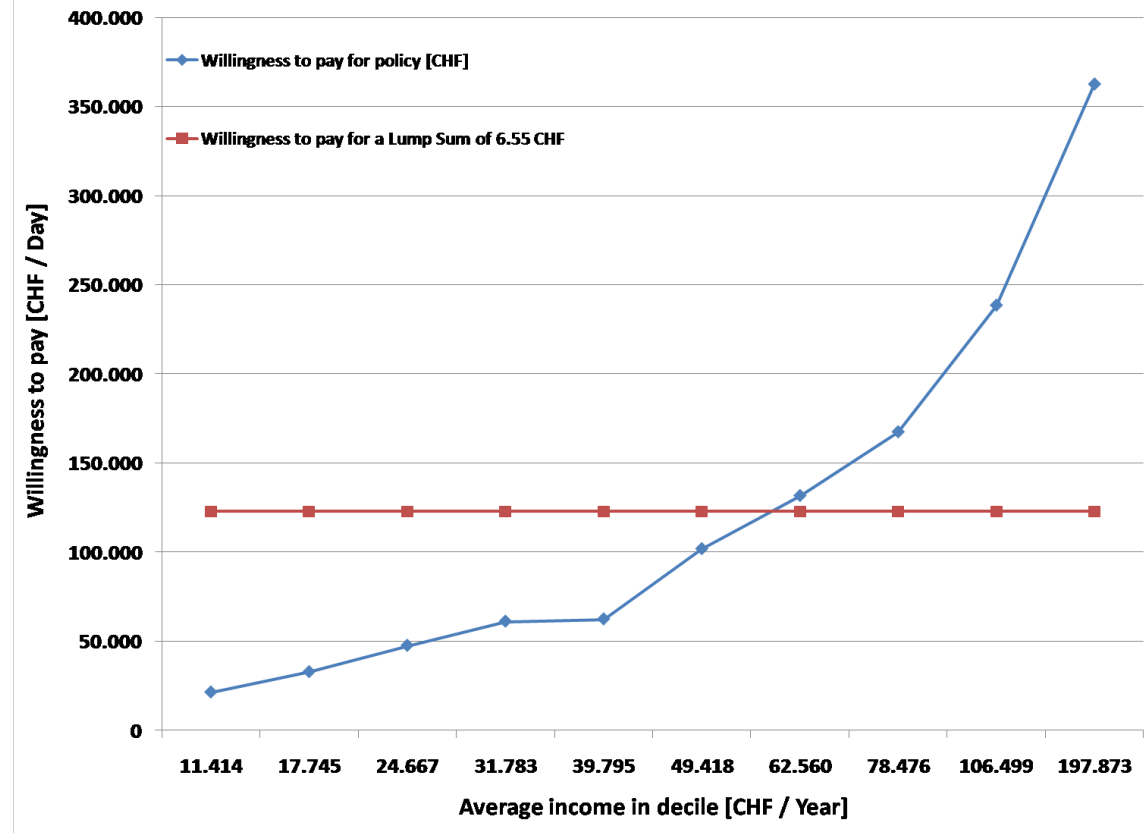

(d) Combined measure: daily willingness to pay

FIGURE 2 Results Zurich Scenario over decils of population sorted by income (c) Average utility changes (d) Daily willingness to pay for the policy change 
At a quick glance one can observe that with increasing income, more persons switch from car to pt. More precisely one can see a break in the increasing pt shares in the 5th decile, where only $1.5 \%$ change mode while in the $3 \mathrm{rd}$ and 4 th decile $1.7 \%$ change mode. Apart from this outlier the mode choice reflects the decreasing importance of travel costs compared to travel time savings when income increases as specified in (5). This is more obvious than in the test scenario where the strongest mode reaction to the measure takes place in the 5th and 6th decile. Thereby the initial distribution of mode choice over income decils should be taken into account. The test scenario's distribution is rather artificial ranging from $100 \%$ car users in the first decil to $100 \%$ public transit users in the 10th decil. Exalting the incentive to switch from car to public transit for people already using this mode can not show any effect in low/high income deciles. For this purpose the more uniform distribution of mode choice of the Zurich scenario is a more suitable starting point.

Increasing utility gains of agents with higher income can also be seen in Fig. 2c that depicts the average utility change of each population decile sorted by income. Each dot is located in the middle of the decile and represents the average utility change per decile. For representation purposes the dots are connected with lines. Obviously, one recognizes raising utility gains with increasing income. In terms of utils, the slope of the curve is slightly positive. The subsequent section shows that this increase has even stronger effects when converting utils into money terms.

\section{DISCUSSION}

For the combined policy case in the large-scale Zurich scenario, a basic analysis of welfare changes along deciles of the population is discussed. The overall effect is calculated by the mean utility gain in the deciles $\overline{\Delta U}_{d}$ (in terms of money) times the (always equal) number of persons in each decile $n$. According to (5), conversion from utility units into CHF is based on individual income $y_{j}$ and utility changes $\Delta U_{j}$ :

$$
\overline{\Delta U}_{d}=\frac{1}{n} \sum_{j=1}^{n} \frac{\Delta U_{j} \cdot y_{j}}{4.58}
$$

Summing this over all ten deciles, the welfare effect of this policy is about 1.23 million CHF per day or almost 300 million CHF per year for the computed 10\% sample of the Zurich metropoltitan population (see section "Zurich, Network and Population"). Thus, following standard economic appraisal methods, the policy should be introduced if this benefit overweights economic costs.

Fig. 2d shows in blue the total daily monetarized gains over deciles of the population, sorted by income. The monetarized gains in every decile can be interpreted as the total willingness to pay for the measure. The red curve tries to explain implementation problems due to low acceptance within the society. If, in a hypothetical case, the same daily welfare gains of 1.23 million CHF were distributed as a monetary lump-sum payment to every member of the population, every person would gain $6.55 \mathrm{CHF}$ per day or every decile 123'000 CHF. This highlights an important implementation problem of policy measures in democratically organized societies: almost $70 \%$ of the population would be better off with the lump-sum payment than with the implementation of the measure and are therefore likely to refuse the latter. Thus, if the simulation results are correct, financing this measure with tax revenues would be more appropriate, assuming a progressive income tax. Whereas financed by non differentiated user fees, this measure would have regressive impact on the income distribution. 
This example is meant to show some possibilities of economic policy evaluation that are feasible with multi-agent microsimulations. Agents optimise their daily plans with respect to individual preferences such as individual income or activity location. Still, there are three main issues that should be addressed in the future: first, for more reliable results, the survey should be designed in a way that all needed parameters can be estimated independently. Second, public transit is assumed to be $100 \%$ reliable, and no fluctuations due to geographic location or line cycles are considered. In principle, using multi-agent transport simulations, makes it possible to combine multiple demographic attributes of the population of interest, e.g. by viewing the geospatial distribution of winners and losers of a measure (5). Neither the measure of this paper nor the public transit simulation features geospatial diversity. Thus analysis in the geographic dimension is strongly homogeneous and a spatial pattern is not visible. In case of a policy that is targeted on some geospatial impact the multi-agent approach should give interesting insights into geospatial distribution of gains and losses (31). Third, utility changes within the simulation are influenced by stochastic effects in the plan selection process, especially for people that switch mode. Nonetheless, it is shown that with this multi-agent approach, welfare computations and equity analysis can be done on the desired level of (dis)aggregation.

\section{CONCLUSION}

Standard economic policy evaluation allows the realisation of projects if the aggregated economic benefit overweights their costs. In democratically organized societies, the implementation of measures with regressive effects on the welfare distribution tends to be complicated due to low public acceptance.

The microscopic simulation approach presented in this paper is capable to help designing better solutions in such situations. In particular, it is shown that income can be included in utility calculations for a better understanding of problems linked to acceptability. Then, in contrast to project evaluation applied in practice, choice modeling and economic evaluation are implemented in a consistent framework since the simulation values are directly used for evaluation. Furthermore, and going beyond (1), it is shown that the approach works in a large-scale real world example for which economic benefits are calculated.

\section{ACKNOWLEDGMENTS}

This work was funded in part by the "Bundesministerium für Bildung und Forschung" (BMBF) within the research project "Adaptive Verkehrssteuerung" (Advest), and in part by the "German Research Foundation" (DFG) within the research project "Detailed evaluation of transport policies using microsimulation". Our compute cluster is maintained by the Department of Mathematics at TU Berlin.

\section{REFERENCES}

1. J.P. Franklin. The distributional effects of transportation policies: The case of a bridge toll for Seattle. PhD thesis, University of Washington, Seattle, 2006.

2. D. Pearce and C.A. Nash. Social appraisal of projects: A text in cost-benefit analysis. Wiley \& Sons, London, 1981.

3. K. Meister, M. Rieser, F. Ciari, A. Horni, M. Balmer, and K.W. Axhausen. Anwendung eines 
agentenbasierten Modells der Verkehrsnachfrage auf die Schweiz. In Proceedings of Heureka '08, Stuttgart, Germany, March 2008.

4. K. Nagel, D. Grether, U. Beuck, M. Rieser, Y. Chen, and K.W. Axhausen. Multi-agent transport simulations and economic evaluation. Journal of Economics and Statistics (Jahrbücher für Nationalökonomie und Statistik), 228(2+3):173-194, 2008. Special issue on agent based models for economic policy advice, edited by B. LeBaron and P. Winker.

5. D. Grether, Y. Chen, M. Rieser, U. Beuck, and K. Nagel. Emergent effects in multi-agent simulations of road pricing. In Annual meeting of the European Regional Science Association ERSA '08, 2008.

6. J. Bates. Economic evaluation and transport modelling: Theory and practice. In K.W. Axhausen, editor, Moving through nets: The physical and social dimensions of travel, chapter 10, pages 279-351. Elsevier, 2006.

7. G. de Jong, A. Daly, E. Kroes, and T. van der Hoorn. Using the logsum in project appraisal. In Proceedings of the meeting of the International Association for Travel Behavior Research (IATBR), Kyoto, Japan, 2006. See www.iatbr.org.

8. B. Raney and K. Nagel. An improved framework for large-scale multi-agent simulations of travel behavior. In Rietveld et al. (32), pages 305-347.

9. M. Balmer, B. Raney, and K. Nagel. Adjustment of activity timing and duration in an agentbased traffic flow simulation. In H.J.P. Timmermans, editor, Progress in activity-based analysis, pages 91-114. Elsevier, Oxford, UK, 2005.

10. N. Lefebvre and M. Balmer. Fast shortest path computation in time-dependent traffic networks. In Proceedings of Swiss Transport Research Conference (STRC) (33). See www.strc.ch.

11. M. Rieser, D. Grether, and K. Nagel. Adding mode choice to a multi-agent transport simulation. Technical Report 09-2758, 2009.

12. C. Gawron. Simulation-based traffic assignment. PhD thesis, University of Cologne, Cologne, Germany, 1998.

13. N. Cetin, A. Burri, and K. Nagel. A large-scale agent-based traffic microsimulation based on queue model. In Proceedings of Swiss Transport Research Conference (STRC) (33). See www.strc.ch. Earlier version, with inferior performance values: Transportation Research Board Annual Meeting 2003 paper number 03-4272.

14. D.E. Kaufman, K.E. Wunderlich, and R.L. Smith. An iterative routing/assignment method for anticipatory real-time route guidance. Technical Report IVHS Technical Report 91-02, University of Michigan Department of Industrial and Operations Engineering, Ann Arbor MI, May 1991.

15. J. Bottom. Consistent anticipatory route guidance. $\mathrm{PhD}$ thesis, Massachusetts Institute of Technology, Cambridge, MA, 2000. 
16. W. S. Vickrey. Congestion theory and transport investment. The American Economic Review, 59(2):251-260, 1969.

17. R. Arnott, A. de Palma, and R. Lindsey. Economics of a bottleneck. Journal of Urban Economics, 27(1):111-130, 1990.

18. D. Charypar and K. Nagel. Generating complete all-day activity plans with genetic algorithms. Transportation, 32(4):369-397, 2005.

19. B. Raney and K. Nagel. An improved framework for large-scale multi-agent simulations of travel behaviour. In Rietveld et al. (32), page 42.

20. D. Grether, B. Kickhöfer, and K. Nagel. Policy evaluation in multi-agent transport simulations considering income-dependent user preferences. In IATBR 2009 conference proceedings, 2009.

21. J.A. Herriges and C.L. Kling. Nonlinear income effects in random utility models. The Review of Economics and Statistics, 81(1):62-72, 1999.

22. K. M. Kockelman. A model for time- and budget-constrained activity demand analysis. Transportation Research Part B: Methodological, 35(3):255-269, 2001.

23. J. Bates. Measuring travel time values with a discrete choice model: A note. Economic Journal, 97(386):493-98, June 1987.

24. B. Kickhöfer. Die Methodik der ökonomischen Bewertung von Verkehrsmaßnahmen in Multiagentensimulationen. Master's thesis, TU Berlin, 2009.

25. S. Jara-Díaz, M. Munizaga, P. Greeven, R. Guerra, and K.W. Axhausen. Estimating the value of leisure from a time allocation model. Transportation Research B, 42(10):946-957, 2008.

26. R. Cervero. Transit pricing research. Transportation, 17:117-139, 1990.

27. M. Vrtic, N. Schüssler, A. Erath, M. Bürgle, K.W. Axhausen, E. Frejinger, M. Bierlaire, R. Rudel, S. Scagnolari, and R. Maggi. Einbezug der Reisekosten bei der Modellierung des Mobilitätsverhaltens. Schriftenreihe 1191, Bundesamt für Strassen, UVEK, Bern, CH, 2008. final report for project SVI 2005/004.

28. Y. Chen, M. Rieser, D. Grether, and K. Nagel. Improving a large-scale agent-based simulation scenario. VSP Working Paper 08-15, VSP, TU Berlin, 2008. See www.vsp.tuberlin.de/publications.

29. SFSO. Eidgenössische Volkszählung. Swiss Federal Statistical Office, Neuchatel, 2000.

30. SFSO. Ergebnisse des Mikrozensus 2005 zum Verkehr. Swiss Federal Statistical Office, Neuchatel, 2006.

31. M. Rieser and K. Nagel. Combined agent-based simulation of private car traffic and transit. In IATBR 2009 conference proceedings, 2009. 
32. P. Rietveld, B. Jourquin, and K. Westin, editors. Towards better performing European Transportation Systems. Routledge, London, 2006.

33. Proceedings of Swiss Transport Research Conference (STRC), Monte Verita, CH. See www.strc.ch. 